\title{
Rapid Incoherent Control of A Simple Quantum System via Two-Step Optimization*
}

\author{
Dong Daoyi ${ }^{1,2}$, Lam James $^{2}$ \\ 1. Key Laboratory of Systems and Control, Institute of Systems Science, Academy of Mathematics and Systems Science, \\ Chinese Academy of Sciences, Beijing 100190, P.R. China \\ E-mail: dydong@amss.ac.cn \\ 2. Department of Mechanical Engineering, The University of Hong Kong, Hong Kong, P.R. China. \\ E-mail: james.lam@hku.hk
}

\begin{abstract}
A rapid incoherent control scheme for driving a simple qubit system from an initial mixed state to an arbitrary target pure state is proposed. This scheme consists of two main steps: rapid purification of the initial mixed state and time optimal transition from the conditional state to the target state. The first step rapidly purifies the initial mixed state into an almost-pure state of the simple qubit system through continuous measurements and feedback control by the protocol proposed in Jacobs 2003 Physical Review A 67 030301(R). The second step finds a set of suitable time optimal controls to drive the simple qubit system from the conditional state to the target state. The switching time between the first step and the second step is determined by the expected purity and the resultant state is an almost-pure state with a high fidelity with the target state. This rapid incoherent control scheme provides an alternative quantum engineering strategy for the rapid preparation of arbitrary pure states of a simple qubit system.
\end{abstract}

Key Words: Incoherent control, Qubit system, Two-step optimization, Rapid purification.

\section{INTRODUCTION}

Quantum information processing technology has drawn much attention of many scientists ${ }^{[1]}$ due to its more powerful ability than classical information processing in some aspects such as factorizing large numbers ${ }^{[2]}$, searching unsorted databases ${ }^{[3]}$ and teleporting unknown quantum states ${ }^{[4]}$. To accomplish some quantum information processing tasks, the quantum systems as the information carrier must be prepared in pure states ${ }^{[5]}$. However, quantum systems often exist naturally in mixed states due to the environmental noise, and decoherence becomes one main obstacle to the practical quantum information processing. One solution is to develop the general principles of quantum control theory to drive quantum systems from an initial state (mixed state or pure state) to a pre-determined target pure state ${ }^{[6,7]}$. In this paper we will consider the preparation of the pure states of quantum systems through a control strategy via a two-step optimization and only focus on the simple qubit system.

In quantum control theory, an important task is the design of control strategies ${ }^{[8]}$. Coherent control strategy is a widely used control method where one manipulates the state of a quantum system by applying semiclassical potentials in a fashion that preserves quantum coherence ${ }^{[9]}$. In fact, the early paradigm of quantum control is mainly open loop coherent control ${ }^{[10,11]}$, which has successfully been used to the quantum control of chemical reaction ${ }^{[12,13]}$. Quantum coherence is a dominant effect in quantum domain, however, quantum measurements usually destroy the coherent characteristics and are often taken as a nuisance for accomplishing some quantum control tasks. Fortunately recent results have shown that quantum measurements can be combined with

${ }^{*}$ This work was supported in part by the National Natural Science Foundation of China under Grant No. 60703083, the K. C. Wong Education Foundation (Hong Kong) and the China Postdoctoral Science Foundation (20060400515). unitary operators to complete some quantum manipulating tasks and enhance the capability of quantum control ${ }^{[14-25]}$. For example, Jacobs ${ }^{[25]}$ used continuous measurements and feedback control to increase the speed of the preparation of the pure states and Pechen et al. ${ }^{[16]}$ investigated nonselective von Neumann measurements for the control of a two-level system. Since quantum measurements destroy quantum coherence, we call this class of control schemes "incoherent control" ${ }^{[22]}$. In this paper, we will focus on the incoherent control strategy and propose a rapid incoherent control scheme based on continuous measurements and feedback control. Our goal is to drive a single qubit from a completely mixed state to an arbitrary target pure state in a time optimal manner. Firstly we rapidly purify the initial mixed state into an almost-pure state (conditional state) through continuous measurements and feedback control using the rapid purification protocol proposed by Jacobs ${ }^{[23]}$. If we select $\sigma_{z}$ as the measurement basis, ideally the conditional state will be an eigenstate of $\sigma_{z}$. However the time required may be too long. Since we expect to rapidly prepare the target state, it may be necessary to select an appropriate time to turn to the second step with the cost of some purity. The second step finds a set of suitable time optimal controls to drive the single qubit from the conditional state to the near-target state. The switching time between the first step and the second step is determined by the expected purity. As an example, this scheme is used to the control design of a spin $1 / 2$ particle driven by a magnetic field.

This paper is organized as follows. Section 2 introduces the rapid purification protocol of a single qubit by weak measurements and Hamiltonian feedback as the first step of the proposed scheme. In Section 3, the time optimal control from the conditional state to the near-target state is designed for a two-level system via a corresponding relation and the analytical expression of minimal time is given. Section 4 discusses some related problems and conclusions are given 
in Section 5.

\section{RAPID PURIFICATION OF A QUBIT SYS- TEM BY CONTINUOUS MEASUREMENTS AND FEEDBACK CONTROL}

The goal of our rapid incoherent control scheme is to drive a simple qubit system from a completely mixed state $\rho_{0}$ to a given pure state $\rho_{T}=\left|\psi_{T}\right\rangle\left\langle\psi_{T}\right|$. To accomplish this task, we firstly select a suitable observable and project the qubit into an eigenstate. This process is called as the purification of $\rho_{0}$ and can be realized by von Neumann measurements or continuous measurements. Von Neumann measurements are treated as instantaneous and are reasonable when the strength of measurements is large enough and the measurement time-scale is much shorter than all other related timescales for a given task ${ }^{[25]}$. Recent results have shown that the continuous measurements of individual quantum systems become possible ${ }^{[26]}$ and the evolution of a quantum system under continuous measurements can be described by a master equation ${ }^{[27]}$. Here we use continuous measurements to purify the initial mixed state. Since the purification process of a quantum system commonly takes some time, the information in the measurement record can be used to change the process of purification via feedback control ${ }^{[28]}$. Jacobs ${ }^{[23]}$ has shown that the use of Hamiltonian feedback control can increase the speed of purification with a factor of two over the no-feedback protocol for the purification of a completely mixed qubit state. Besides the interest of quantum phenomena, this discovery is also helpful for rapid state preparation since the rapid initialization of quantum logic units is very significant for practical quantum information processing. Hence we adopt Jacobs' rapid purification protocol as the first step of the present control strategy.

In the first step, if we do not consider the Hamiltonian action, the evolution equation of the density matrix $\rho$ of the qubit system under the continuous measurement of $\sigma_{z}$ can be described as follows ${ }^{[23]}$ :

$$
d \rho=-\kappa\left[\sigma_{z},\left[\sigma_{z}, \rho\right]\right] d t+\sqrt{2 \kappa}\left(\sigma_{z} \rho+\rho \sigma_{z}-2\left\langle\sigma_{z}\right\rangle \rho\right) d W
$$

where $\kappa$ is a positive constant called measurement strength, and $d W$ is a Wiener increment with zero mean and variance equal to $d t$. We set $\hbar=1$ and do not consider the Hamiltonian evolution of systems. Equation (1) is called as the stochastic master equation (SME). The continuous measurement will eventually project the qubit onto one $\left(\rho_{e}^{0}=|0\rangle\langle 0|\right.$ or $\rho_{e}^{1}=|1\rangle\langle 1|$ ) of the eigenstates of $\sigma_{z}$.

For the state $\rho$ of a qubit system, we can represent it in terms of the Bloch vector $\boldsymbol{r}=(x, y, z)=$ $\left(\operatorname{Tr}\left\{\rho \sigma_{x}\right\}, \operatorname{Tr}\left\{\rho \sigma_{y}\right\}, \operatorname{Tr}\left\{\rho \sigma_{z}\right\}\right)$

$$
\rho=\frac{1}{2}(I+\boldsymbol{r} \cdot \sigma)
$$

where $I$ is $2 \times 2$ identical matrix, $\sigma$ denotes the Pauli matrices $\sigma=\left(\sigma_{x}, \sigma_{y}, \sigma_{z}\right)$ and

$$
\sigma_{x}=\left(\begin{array}{cc}
0 & 1 \\
1 & 0
\end{array}\right) \quad, \quad \sigma_{y}=\left(\begin{array}{cc}
0 & -i \\
i & 0
\end{array}\right) \quad, \quad \sigma_{z}=\left(\begin{array}{cc}
1 & 0 \\
0 & -1
\end{array}\right) .
$$

The SME equation can be rewritten as ${ }^{[25]}$

$$
\mathrm{d} x=-(4 \kappa d t+z \sqrt{8 \kappa} \mathrm{d} W) x
$$

$$
\begin{aligned}
\mathrm{d} y & =-(4 \kappa d t+z \sqrt{8 \kappa} \mathrm{d} W) y \\
\mathrm{~d} z & =\left(1-z^{2}\right) \sqrt{8 \kappa} \mathrm{d} W
\end{aligned}
$$

Therefore, the state space of a qubit system corresponds to the Bloch sphere, where pure states lie on the surface of the sphere ( $\rho_{e}^{0}$ corresponding to the North pole and $\rho_{e}^{1}$ to the South pole) and mixed states are in the interior of the sphere. The purity of a state $\rho$ corresponds to the squared length of the Bloch vector. The impurity of the qubit state can be quantified by the following equation ${ }^{[23]}$ :

$$
L=1-\operatorname{Tr}\left[\rho^{2}\right]=\frac{1}{2}\left(1-x^{2}-y^{2}-z^{2}\right)
$$

$L=0$ for all pure states and $L=0.5$ for the completely mixed state. The goal of the first step is to pull an interior state $\rho_{0}$ towards one $\left(\rho_{e}^{0}=|0\rangle\langle 0|\right.$ or $\left.\rho_{e}^{1}=|1\rangle\langle 1|\right)$ of the poles, i.e. purification of $\rho_{0}$. Assume we expect to purify $\rho_{0}$ into $\rho_{e}^{0}$ and this can be accomplished by Jacobs' protocol ${ }^{[23]}$. To rapidly purify a mixed state, the Bloch vector of the qubit is rotated onto the $x-y$ plane (perpendicular to the measurement axis $z$ ) based on the measurement record after each measurement step. In fact, the protocol is optimal in the sense of maximizing the fidelity of the qubit with some fixed pure state at a given time ${ }^{[28]}$, which has been rigorously proven by Wiseman and Bouten ${ }^{[31]}$ by applying simple concepts from optimal control theory. The detailed protocol for rapid state purification can be found in ${ }^{[23,25]}$. It should be pointed out that usually we can rapidly pull the initial completely mixed state into an almost-pure state by Jacobs' protocol. However, the goal of the first step in our incoherent control scheme is to drive the initial state into the eigenstate $\rho_{e}^{0}$. In some practical applications, the almost-pure state may be enough, so we can select $\rho_{e}^{0}$ as the estimation of the conditional state for the control design of the second step. The start time of the second step can be determined through the expected purity. Assume the expected purity is $1-\epsilon(\epsilon \ll 1)$ and the measurement strength is set as $\kappa=1 / 2$, the time required is ${ }^{\text {[24] }}$

$$
t=-\frac{1}{4} \ln 2 \epsilon
$$

Since Jacob's rapid purification protocol is a deterministic scheme ${ }^{[23]}$, the switching time can be precisely determined according to equation (8) in principle.

\section{TIME OPTIMAL TRANSITION OF THE CONDITIONAL STATE}

After the initial mixed state is rapidly projected using Jacob's rapid purification protocol, the conditional state becomes an almost-pure state which has a high fidelity $1-\epsilon$ with the eigenstate $\rho_{e}^{0}$. Since we take $\rho_{e}^{0}$ as an estimation of the conditional state, the control design for rapidly transferring the conditional state into the target state $\rho_{T}$ can approximately be accomplished by the time optimal control design from $\rho_{e}^{0}$ to $\rho_{T}=\left|\psi_{T}\right\rangle\left\langle\psi_{T}\right|$. In quantum control, the transition from the pure state $\rho(0)=|\psi(0)\rangle\langle\psi(0)|$ to the pure state $\rho(t)=|\psi(t)\rangle\langle\psi(t)|$ can be accomplished through a unitary transformation $U(t)$ whose time evolution is determined by the time-dependent Schrödinger equation (setting $\hbar=1$ )

$$
i \dot{U}(t)=H(t) U(t), \quad U(0)=I
$$


where $H(t)$ is the Hamiltonian of the quantum system. In this paper we only concentrate on two-dimensional quantum systems, i.e. simple qubit systems, and $H(t), U(t)$ are $2 \times 2$ matrices. Obviously

$$
\rho(t)=U(t) \rho(0) U^{\dagger}(t)
$$

Therefore the control of pure states can be converted into the design problem of $U(t)$. To actively accomplish the task, we should introduce external control $\left\{u_{j}(t)\right\}$ into $H(t)$ and in many situations it can be expressed as

$$
H(t)=H_{0}(t)+H_{I}(t)=H_{0}(t)+\sum_{j=1}^{n} u_{j}(t) H_{j}
$$

where $H_{0}$ is the internal Hamiltonian and $H_{I}(t)$ is the control Hamiltonian.

Some researchers have studied the time optimal control problem of the above model (9)-(11). For example, Khaneja et $a l^{[32]}$ present the time optimal control with unbounded control. Boscain and his co-workers ${ }^{[33,34]}$ studied the time optimal control of population transfer of two- and three-level quantum systems with bounded control. It is difficult to derive the general results for the time optimal control of quantum systems. To demonstrate our incoherent control scheme in this paper, we will only concentrate on a two-level quantum system driven by a real unbounded control used in [32]. This system describes the evolution of the $z$-component of the spin of a spin $1 / 2$ particle driven by a magnetic field. The field is constant along the $z$ axis and can produce a rapid $x$ rotation on the spin by radio-frequency (rf) pulses. The unitary evolution of this single-spin system can be given

$$
i \dot{U}(t)=\left[I_{z}+u I_{x}\right] U(t), \quad U(0)=I
$$

where the control $u \in \mathbb{R}$ and

$$
\begin{gathered}
I_{x}=\frac{1}{2} \sigma_{x}=\frac{1}{2}\left(\begin{array}{cc}
0 & 1 \\
1 & 0
\end{array}\right) \\
I_{z}=\frac{1}{2} \sigma_{z}=\frac{1}{2}\left(\begin{array}{cc}
1 & 0 \\
0 & -1
\end{array}\right)
\end{gathered}
$$

It is easy to check the controllability condition of the model (12) and this two-level system is completely controllable on the Bloch sphere ${ }^{[7]}$.

If we ignore the global phase, any two pure states $\rho_{1}=$ $\left|\psi_{1}\right\rangle\left\langle\psi_{1}\right|$ and $\rho_{2}=\left|\psi_{2}\right\rangle\left\langle\psi_{2}\right|$ of a qubit system can be described as

$$
\begin{gathered}
\rho_{1}=\left(\begin{array}{cc}
\cos ^{2} \frac{\theta_{1}}{2} & e^{-i \varphi_{1}} \cos \frac{\theta_{1}}{2} \sin \frac{\theta_{1}}{2} \\
e^{i \varphi_{1}} \cos \frac{\theta_{1}}{2} \sin \frac{\theta_{1}}{2} & \sin ^{2} \frac{\theta_{1}}{2}
\end{array}\right) \\
\rho_{2}=\left(\begin{array}{cc}
\cos ^{2} \frac{\theta_{2}}{2} & e^{-i \varphi_{2}} \cos \frac{\theta_{2}}{2} \sin \frac{\theta_{2}}{2} \\
e^{i \varphi_{2}} \cos \frac{\theta_{2}}{2} \sin \frac{\theta_{2}}{2} & \sin ^{2} \frac{\theta_{2}}{2}
\end{array}\right)
\end{gathered}
$$

where $\theta_{1}, \theta_{2} \in[0, \pi]$ and $\varphi_{1}, \varphi_{2} \in[0,2 \pi]$. Denote $U_{F}$ as the unitary transformation that can accomplish the control task in the second step and let $\rho_{1}=\rho_{e}^{0}, \rho_{2}=\rho_{T}$, thus $\rho_{T}=U_{F} \rho_{e}^{0}$.

For the time optimal control of the spin $1 / 2$ system, if the control may be unbounded, we can easily obtain the following theorem.
Theorem 1 For the system (12), given any target unitary transformation $U_{F} \in S U(2)$, there exists a unique $\beta \in[0, \pi]$ such that $U_{F}=\exp \left(-i \alpha I_{x}\right) \exp \left(-i \beta I_{z}\right) \exp \left(-i \gamma I_{x}\right)$, where $\alpha, \gamma \in \mathbb{R}$, and the minimum time for accomplishing $U_{F}$ is $\beta$ and satisfies $\beta=\left(\arccos \left(\sin \theta_{2} \cos \varphi_{2}\right)-\right.$ $\left.\arccos \left(\sin \theta_{1} \cos \varphi_{1}\right)\right) \bmod (\pi)$.

In Theorem $1 S U(2)$ denotes the two-dimensional special unitary group and $U_{F}$ consists of three rotations $U_{x}(\alpha)=$ $\exp \left(-i \alpha I_{x}\right), U_{z}(\beta)=\exp \left(-i \beta I_{z}\right), U_{x}(\gamma)=\exp \left(-i \gamma I_{x}\right)$. Since the control $u$ may be unbounded, the time required to generate $U_{x}(\alpha)=\exp \left(-i \alpha I_{x}\right)$ and $U_{x}(\gamma)=\exp \left(-i \gamma I_{x}\right)$ is zero. Hence all points in the corresponding circle of $U_{x}(\alpha)=\exp \left(-i \alpha I_{x}\right)\left(\right.$ or $\left.U_{x}(\gamma)=\exp \left(-i \gamma I_{x}\right)\right)$ are equivalent from the time optimization perspective. So it is slightly different from Theorem 1 in [32], where they argue that there exists a unique $\beta \in[0,2 \pi]$. We can easily give the analytical expression of $\beta$ according to the geometrical relations:

\section{$\beta=$}

$\left(\arccos \left(\sin \theta_{2} \cos \varphi_{2}\right)-\arccos \left(\sin \theta_{1} \cos \varphi_{1}\right)\right) \bmod (\pi)$

Since the specific values of $\alpha$ and $\gamma$ are not unique, we only consider the value $\beta$ for two special cases. Obviously $\varphi_{1}=$ 0 and $\theta_{1}=0$. When the target state is

$$
\rho_{T 1}=\frac{1}{2}\left(\begin{array}{cc}
1 & -i \\
i & 1
\end{array}\right)
$$

the minimal time is

$$
\beta_{1}=0
$$

When the target state is

$$
\rho_{T 2}=\frac{1}{4}\left(\begin{array}{cc}
3 & \sqrt{3} \\
\sqrt{3} & 1
\end{array}\right)
$$

the minimal time is

$$
\beta_{2}=\frac{\pi}{3}
$$

Hence we can give the sketch as shown in Fig. 1 for the evolution of the qubit under this incoherent control strategy. From the above results, we can find that the minimal time required from $\rho_{e}^{0}$ to $\rho_{T 1}$ is zero because the task can be accomplished only using the rotation $U_{x}$ which need no time under the unbounded assumption of $u$. Moreover it is obvious that the different conditional states do not effect the minimal time since the transition between two eigenstates can be accomplished only using the rotation $U_{x}$. Here we assume that we rapidly purify the initial state into an almost-pure state near to $\rho_{e}^{0}$ in the first step. For the case $\rho_{e}^{1}$, we can also obtain analogous results.

\section{DISCUSSIONS}

In quantum control, the quantum measurement is regarded as a nuisance in some situations to accomplish some quantum control tasks since it destroys the coherence of measured quantum systems. However, some recent results have shown that the quantum measurement on the controlled system or the ancillary system can improve the controllability of quantum systems ${ }^{[14-22]}$ or enhance the capability of quantum control ${ }^{[15,16,36]}$. For example, Roa et al. ${ }^{[17]}$ applied sequential measurements of two noncommuting observables to 


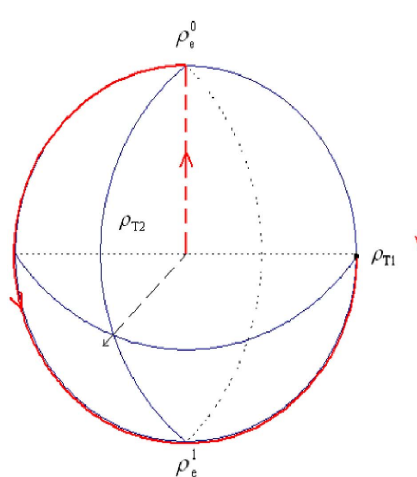

(a)

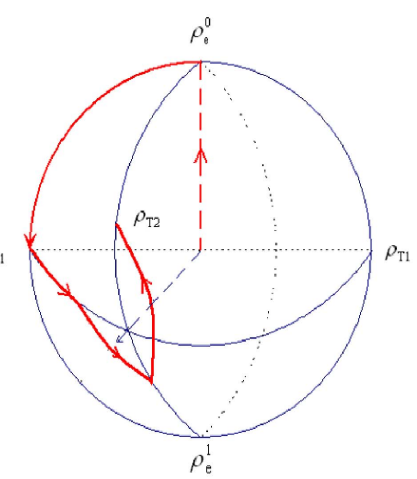

(b)
Fig. 1 The sketch for the evolution process of a simple qubit system under the rapid incoherent control strategy. (a) The target state is $\rho_{T 1}$; (b) The target state is $\rho_{T 2}$.

drive an unknown mixed quantum state to a known pure state without the use of unitary transformation. Pechen et al. ${ }^{[16]}$ explored nonselective von Neumann measurements to control quantum dynamics. Romano and D'Alessandro ${ }^{[22]}$ investigated accessibility and controllability of a quantum system coupled to a quantum probe under the hypothesis that the external control affects only the probe. In the cited work, quantum measurements commonly are von Neumann measurements. In this paper we explore the role of continuous measurements combined with unitary transformations to the control of quantum systems. In the present control scheme, the continuous measurement is regarded as a kind of effective control and it rapidly projects the initial mixed state into an almost-pure state with the help of Hamiltonian feedback. Our scheme includes two main steps: the purification of the initial mixed state and the transition from the conditional state to the target state. Since the aim of the first step is to project the initial state to an eigenstate, we can select a general continuous measurement without the following of feedback control. The Jacob's rapid purification protocol is not necessary for our scheme and we use the protocol only for the rapid control. Besides the protocol proposed by Jacobs, Wiseman and Ralph ${ }^{[24]}$ have also proposed another rapid purification protocol to minimize the average time required for a qubit to reach a given purity ${ }^{[24]}$, which can also be used to the present incoherent control scheme without intrinsic change. For $N$ dimensional systems, Combes and Jacobs ${ }^{[37]}$ have shown that there exists an analogous feedback algorithm which will speed up the rate of state purification by at least a factor of $2(N+1) / 3$. Hence the combination of continuous measurements and Hamiltonian feedback will be more attractive for complex quantum systems. Moreover, these strategies have been used to some specific quantum systems such as Cooper pair boxes ${ }^{[28]}$ and entangled bipartite quantum systems ${ }^{[38]}$, which provides a supportive evidence for the realizability of our incoherent control scheme. In the general quantum control problems, one commonly assumes that the initial state is known. However this is not practical for many actual tasks. In this paper we can also assume that the initial state is unknown which is a more natural assumption. Different from the measurement-driven evolution scheme ${ }^{[17]}$ by sequential measurements of two noncommuting observables, the present strategy needs only the mea- surements of an observable. Moreover we also consider the optimality of control time. It is worth noting that the two steps in our scheme are time optimal, respectively, however, it does not mean that our rapid incoherent control scheme is necessarily the globally time optimal strategy for accomplishing this class of control tasks. More optimal strategies such as energy optimal problem and globally optimal scheme should be further explored, and the present scheme can also be extended to some more complex systems. Moreover, the present incoherent control scheme is a theoretical design strategy and it is realizable in principle. The experimental implementations maybe need some slight changes as that of Cooper pair boxes ${ }^{[28]}$.

\section{CONCLUSION}

With the development of quantum technology, the control of quantum systems has become an important task in quantum information science, cold atom physics and quantum optics. The early work mainly focuses on the coherent control strategy. Recently the incoherent control has drawn the attention of some researchers. In this paper we propose a rapid incoherent control scheme to accomplish some quantum control tasks. This scheme mainly consists of two steps: rapid purification of the initial mixed state and time optimal transition from the conditional state to the target state. The first step is accomplished by weak measurements and feedback control, and the second step is realized through coherent manipulations. This incoherent control scheme provides an alternative quantum engineering strategy for the rapid preparation of arbitrary pure states of a simple qubit system and can also be extended to multi-qubit systems.

\section{ACKNOWLEDGMENT}

D.Y. Dong would like to thank Dr. Bo Qi and Dr. Hao Pan for helpful comments and suggestions.

\section{REFERENCES}

[1] NIELSEN M A, CHUANG I L. Quantum Computation and Quantum Information. Cambridge, England: Cambridge University Press, 2000.

[2] SHOR P W. Algorithms for quantum computation: discrete logarithms and factoring. In Proceedings of the 35th Annual Symposium on Foundations of Computer Science, IEEE Press, Los Alamitos, CA, 1994: 124-134.

[3] GROVER L K. Quantum mechanics helps in searching for a needle in a haystack. Physical Review Letters, 1997, 79: 325-327.

[4] BOUWMEESTER D, PAN J W, MATTLE K, EIBL M, WEINFURTER H, ZEILINGER A. Experimental quantum teleportation. Nature, 1997, 390: 575-579.

[5] CIRAC J I, ZOLLER P. Quantum computations with cold trapped ions. Physical Review Letters, 1995, 74: 4091-4094.

[6] DOWLING J P, MILBURN G J. Quantum technology: the second quantum revolution. Philosophical Transactions of the Royal Society of London A, 2003, 361: 1655-1674.

[7] CHEN Z H, DONG D Y, ZHANG C B.Quantum Control Theory: An Introduction. Hefei: University of Science and Technology of China Press, 2005.

[8] RABITZ H, DE VIVIE-RIEDLE R, MOTZKUS M, KOMPA $\mathrm{K}$. Whither the future of controlling quantum phenomena? Science, 2000, 288: 824-828.

[9] LLOYD S. Coherent quantum feedback. Physical Review A, 2000, 62: 022108 . 
[10] SHAPIRO M, BRUMER P. Laser control of product quantum state populations in unimolecular reactions. Journal of Chemical Physics, 1986, 84(7): 4103-4104.

[11] WARREN W S, RABITZ H, DAHLEH M. Coherent control of quantum dynamics: The dream is alive. Science, 1993, 259: 1581-1589.

[12] RICE S A. Interfering for the good of a chemical reaction. Nature, 2001, 409: 422-426.

[13] RICE S A, ZHAO M S. Optical Control of Molecular Dynamics. New York: John Wiley \& Sons, Inc., 2000.

[14] MENDES R V, MAN'KO V I. Quantum control and the Strocchi map. Physical Review A, 2003, 67: 053404.

[15] SHUANG F, PECHEN A, HO T S, RABITZ H. Observationassisted optimal control of quantum dynamics. Journal of Chemical Physics, 2007, 126: 134303.

[16] PECHEN A, IL'IN N, SHUANG F, RABITZ H. Quantum control by von Neumann measurements. Physical Review A, 2006, 74: 052102.

[17] ROA L, DELGADO A, LADRÓN DE GUEVARA M L, KLIMOV A B. Measurement-driven quantum evolution. Physical Review A, 2006, 73: 012322.

[18] ZHANG M, DAI H Y, XI Z R, XIE H W, HU D W. Combating dephasing decoherence by periodically performing tracking control and projective measurement. Physical Review A, 2007, 76: 042335.

[19] XI Z R, JIN G S. Performance comparison between classical and quantum control for a simple quantum system. Physica A, 2008, 387: 1056-1062.

[20] MANDILARA A, CLARK J W. Probabilistic quantum control via indirect measurement. Physical Review A, 2005, 71: 013406.

[21] ROMANO R, D'ALESSANDRO D. Environment-mediated control of a quantum system. Physical Review Letters, 2006, 97: 080402.

[22] ROMANO R, D'ALESSANDRO D. Incoherent control and entanglement for two-dimensional coupled Systems. Physical Review A, 2006, 73: 022323.

[23] JACOBS K. How to project qubits faster using quantum feedback. Physical Review A, 2003, 67: 030301(R).

[24] WISEMAN H M, RALPH J F. Reconsidering rapid qubit purification by feedback. New Journal of Physics,2006, 8: 90.

[25] JACOBS K. Optimal feedback control for rapid preparation of a qubit. Proceedings of SPIE, 2004, 5468:355-364.
[26] GEREMIA J M, STOCKTON J K, MABUCHI H. Real-time quantum feedback control of atomic spin-squeezing. Science, 2004, 304(5668): 270-273.

[27] BOUTEN L, EDWARDS S, BELAVKIN V P. Bellman equations for optimal feedback control of qubit states. Journal of Physics B- Atomic Molecular and Optical Physics, 2005, 38(3): 151-160.

[28] GRIFFITH E J, HILL C D, RALPH J F, WISEMAN H M, JACOBS K. Rapid-state purification protocols for a Cooper pair box. Physical Review B, 2007, 75: 014511.

[29] KOROTKOV A N. Continuous quantum measurement of a double dot. Physical Review B, 1999, 60: 5737-5742.

[30] KOROTKOV A N. Selective quantum evolution of a qubit state due to continuous measurement. Physical Review B, 2001, 63: 115403 .

[31] WISEMAN H M, BOUTEN L. Optimality of feedback control strategies for qubit purification. arXiv quant-ph: 0707.3001, 2007.

[32] KHANEJA N, BROCKETT R, GLASER S J. Time optimal control in spin systems. Physical Review A, 2001, 63: 032308 .

[33] BOSCAIN U, CHARLOT G, GAUTHIER J P, GUÉRIN S, JAUSLIN H R. Optimal control in laser-induced population transfer for two- and three-level quantum systems. Journal of Mathematical Physics, 2002, 43(5): 2107-2132.

[34] BOSCAIN U, MASON P. Time minimal trajectories for a spin 1/2 particle in a magnetic field. Journal of Mathematical Physics, 2006, 47: 062101.

[35] LIBERZON D, MORSE A S. Basic problems in stability and design of switched systems. IEEE Control Systems Magazine, 1999, 19: 59-70.

[36] ZHANG C B, DONG D Y, CHEN Z H. Control of noncontrollable quantum systems: a quantum control algorithm based on Grover iteration. Journal of Optics B: Quantum and Semiclassical Optics, 2005, 7: S313-S317.

[37] COMBES J, JACOBS K. Rapid state reduction of quantum systems using feedback control. Physical Review Letters, 2006, 96: 010504

[38] HILL C, RALPH J F. Weak measurement and rapid state reduction in entangled bipartite quantum systems. New Journal of Physics, 2007, 9: 151. 